ISSN(print): 2643-9840, ISSN(online): 2643-9875

Volume 03 Issue 11 November 2020

DOI: 10.47191/ijmra/v3-i11-01, Impact Factor: 5.522

Page No.- 185-198

\title{
The Dynamics of Differential Impacts of COVID-19 on African Countries Compared to Other Parts of the World
}

\author{
Joseph Oyepata Simeon ${ }^{1}$, Musa Tabitha Lubo ${ }^{2}$, Joseph Opeyemi Tosin $^{3}$, Ibhafidon Irabor ${ }^{4}$ \\ ${ }^{1}$ Department of Pharmacology, Faculty of Pharmaceutical Sciences, Federal University, Oye Ekiti State, Nigeria. \\ ${ }^{2}$ Department of Pharmacognosy, Faculty of Pharmacy, Gombe State University, Gombe State, Nigeria \\ ${ }^{3}$ Department of Pharmacy, University College Hospital, Ibadan, Oyo State, Nigeria \\ ${ }^{4}$ Department of Mathematics, Faculty of Natural and Applied Science, Nasarawa State University, Nasarawa \\ State, Nigeria.
}

\begin{abstract}
Corona virus disease (COVID-19) is an infectious disease caused by a newly discovered corona virus. Most people who fall sick with COVID-19 experience mild to moderate symptoms and recover without special treatment. A number of deaths have been recorded across world. The aim of this study is to investigate and compare the extent the virus affects Africa relative to other parts of the world and it significant. One hundred and eighty seven (187) countries were selected randomly based on their continents and cases of infection. Data from each country were obtained from United Nations Geo scheme and WHO as at October 12, 2020 and were analyzed and compared to that of the United State of America (USA). USA was used as a Comprise Factor (CF) because it has one of the best healthcare system and high COVID-19 cases. Subsequent examination of associations between the proportion of COVID-19 cases, recovery and deaths of each country to the United State of America was carried out. All data used in these analyses are from publicly available data sets. Data analyzed revealed that almost all African countries appears to be least affected by the virus. African countries have the least impacted and lesser number of deaths index. Result from the study suggests that the virus affect Africa the least when compared to USA and other continents in terms of cases and mortality. This may be due to among other factors a more robust immune response. The result also helps to provide relative explanation as to how significant developing and providing vaccine may be to various part of the world.
\end{abstract}

KEYWORD: Africa, USA, COVID-19, Nigeria, infection.

\section{INTRODUCTION}

Corona viruses are a group of RNA viruses that cause diseases in mammals and birds. In humans and birds, they cause respiratory tract infections that can range from mild to lethal ${ }^{1}$. Mild illnesses in humans include some cases of the common cold (which is also caused by other viruses, predominantly rhinoviruses), while more lethal varieties can cause SARS, MERS, and COVID-19 ${ }^{1}$. Corona viruses are members of the subfamily Orthocoronavirinae, in the family Coronaviridae, order Nidovirales, and realm Riboviria ${ }^{1,2}$. They are enveloped viruses with a positive-sense singlestranded RNA genome and a nucleocapsid of helical symmetry ${ }^{2}$. The genome size of coronaviruses ranges from approximately 26 to 32 kilobases, one of the largest among RNA viruses ${ }^{2,3}$. Coronavirus, any virus belonging to the family Coronaviridae. Coronaviruses have enveloped virions (virus particles) that measure approximately $120 \mathrm{~nm}\left(1 \mathrm{~nm}=10^{-9}\right.$ metre) in diameter. Club-shaped glycoprotein spikes in the envelope give the viruses a crownlike, or coronal, appearance. The nucleocapsid, made up of a protein shell known as a capsid and containing the viral nucleic acids, is helical or tubular ${ }^{4,5,6}$. The corona virus genome consists of a single strand of positive-sense RNA (ribonucleic acid). Coronaviridae is generally considered to contain two genera, Corona virus and Toro virus, which differ in nucleocapsid morphology, the former being helical and the latter being tubular $^{7}$. Corona viruses are important agents of gastrointestinal disease in humans, poultry, and bovines. In humans, a species known as SARS corona virus (or Severe acute respiratory syndrome corona virus) causes a highly contagious respiratory disease that is characterized by symptoms of fever, cough, and muscle ache, often with progressive difficulty in breathing. The virus emerged in humans in 2002; it likely jumped to humans from an animal reservoir, believed to be horseshoe bats. ${ }^{8}$. The ability of SARS corona virus to jump to humans undoubtedly required genetic changes in the virus. These changes are suspected to have occurred in the palm civet, since the SARS virus present in horseshoe bats is unable to infect human's directly ${ }^{9}$. Corona viruses are a group of RNA viruses that cause diseases in mammals and birds. In humans and birds, they cause respiratory tract infections that can range from mild to lethal (Wertheim et al., 2013). Mild illnesses in humans include 


\section{The Dynamics of Differential Impacts of COVID-19 on African Countries Compared to Other Parts of the World}

some cases of the common cold (which is also caused by other viruses, predominantly rhinoviruses), while more lethal varieties can cause SARS, MERS, and COVID-19 ${ }^{10}$. In cows and pigs they cause diarrhea, while in mice they cause hepatitis and encephalomyelitis. There are as yet no vaccines or antiviral drugs to prevent or treat human corona virus infections. Corona virus disease (COVID-19) is an infectious disease caused by a newly discovered coronavirus ${ }^{10,11}$.

The COVID-19 pandemic, also known as the corona virus pandemic, is an ongoing pandemic of corona virus disease 2019 (COVID-19) caused by severe acute respiratory syndrome corona virus 2 (SARS-CoV-2) ${ }^{12,13}$. The disease was first identified in December 2019 in Wuhan, China (ICTV, 2019). The World Health Organization declared the outbreak a Public Health Emergency of International Concern on 30 January 2020 and a pandemic on 11 March. As of 17 September 2020, more than 29.9 million cases have been reported in 188 countries and territories, resulting in more than 942,000 deaths; more than 20.3 million people have recovered ${ }^{2,14}$. The COVID-19 virus spreads primarily through droplets of saliva or discharge from the nose when an infected person coughs or sneezes. Many larger droplets rapidly fall to the ground, however some can be suspended in air as aerosols, especially in indoor spaces ${ }^{15}$. It may also be transmitted via contaminated surfaces, although this has not been conclusively demonstrated ${ }^{16,17}$. It can spread for up to two days prior to symptom onset, and from people who are asymptomatic ${ }^{18}$. People remain infectious in moderate cases for 7-12 days, and up to two weeks in severe cases ${ }^{19}$.

The attack rate or transmissibility (how rapidly the disease spreads) of a virus is indicated by its reproductive number (Ro, pronounced R-naught or r-zero), which represents the average number of people to which a single infected person will transmit the virus. Whose estimated (on Jan. 23) Ro to be between 1.4 and 2.5 2,20.

There has been concern about the consequence of the viral outbreak in Africa. The United Nations Economic Commission for Africa (UNECA) had in April predicted that anywhere between 300,000 and 3.3 million African people could lose their lives as a direct result of COVID-19 ${ }^{21}$. The World Health Organization (WHO) warned African countries to "prepare for the worst", while Melinda Gates, co-chair of the Bill \& Melinda Gates Foundation, believed that It was going to be horrible in the developing world. She suggested that part of the reason case numbers don't look very bad is because they don't have access to very many test $^{22}$. The aim of this study is to investigate and compare the extent the virus affects Africa relative to other parts of the world and it significant.

\section{METHOD}

One hundred and eighty seven (187) countries from different continent and regions of the world was randomly selected from each continent and based on COVID-19 cases in each country. The list of countries and territories with their continental regional classification is based on the United Nations Geoscheme and WHO. Sources and data used were provided under Latest Updates from WHO/World meter's on October 12, 2020. Data obtained for each country was analyzed and compared to that of the United State of America (USA). USA was used as a Comparism Factor (CF) because it has one of the best healthcare systems in the world and high COVID-19 cases. Subsequent examination of associations between the proportion of COVID-19 cases, recovery and deaths of each country to the United State of America was carried out. All data used in these analyses are from publicly available data sets.

\section{STATISTICAL ANALYSES}

Parameters such as total incidences/cases, total deaths and total recovered of countries was compared against figures and values obtained for USA. Bivariate analysis was done with Chi-square test to compare proportions for variables. In reporting these results, country-level characteristics are scaled to represent a comparison of two countries similar in all other respects. Thus, rate ratios greater than one mean that higher levels of a given characteristic are associated with higher rates of COVID-19 cases or deaths, while rate ratios less than one mean that lower levels of a given characteristic are associated with lower rates of COVID-19 cases or deaths.

Result

Infectious, recovery and mortality rate of COVID-19 based on country

Data analyzed revealed that with exception of South Africa, Africa appears to be least affected by the virus

Table 1: Infectious, recovery and mortality rate of COVID-19 based on country

\begin{tabular}{|l|l|l|l|l|l|}
\hline & Country, & Total & Total & Total & \multirow{2}{*}{ Population (D) } \\
\hline S/N & Other & Cases (A) & Deaths (B) & Recovered (C) & \\
\hline 1 & World & $37,786,413$ & $1,081,848$ & $28,374,689$ & \\
\hline 2 & USA & $\mathbf{7 , 9 9 2 , 8 1 0}$ & $\mathbf{2 1 9 , 7 0 2}$ & $\mathbf{5 , 1 2 8 , 3 9 0}$ & $331,547,490$ \\
\hline 3 & India & $\mathbf{7 , 1 2 0 , 5 3 8}$ & $\mathbf{1 0 9 , 1 8 4}$ & $\mathbf{6 , 1 4 9 , 5 3 5}$ & $1,383,789,657$ \\
\hline
\end{tabular}


The Dynamics of Differential Impacts of COVID-19 on African Countries Compared to Other Parts of the World

\begin{tabular}{|c|c|c|c|c|c|}
\hline 4 & Brazil & $5,094,979$ & 150,506 & $4,470,165$ & $212,982,744$ \\
\hline 5 & Russia & $1,312,310$ & 22,722 & $1,024,235$ & $145,952,169$ \\
\hline 6 & Colombia & 911,316 & 27,834 & 789,787 & $51,034,004$ \\
\hline 7 & Argentina & 894,206 & 23,868 & 721,380 & $45,311,598$ \\
\hline 8 & Spain & 890,367 & 32,929 & N/A & $46,759,903$ \\
\hline 9 & Peru & 849,371 & 33,305 & 743,969 & $33,099,165$ \\
\hline 10 & Mexico & 817,503 & 83,781 & 594,180 & $129,310,283$ \\
\hline 11 & France & 734,974 & 32,683 & 100,828 & $65,314,276$ \\
\hline 12 & South Africa & 692,471 & 17,780 & 623,765 & $59,516,399$ \\
\hline 13 & UK & 603,716 & 42,825 & N/A & $67,986,204$ \\
\hline 14 & Iran & 500,075 & 28,544 & 406,389 & $84,291,482$ \\
\hline 15 & Chile & 481,371 & 13,318 & 453,352 & $19,162,070$ \\
\hline 16 & Iraq & 402,330 & 9,852 & 336,157 & $40,469,182$ \\
\hline 17 & Bangladesh & 379,738 & 5,555 & 294,391 & $165,147,049$ \\
\hline 18 & Italy & 354,950 & 36,166 & 239,709 & $60,436,599$ \\
\hline 19 & Philippines & 342,816 & 6,332 & 293,152 & $109,985,754$ \\
\hline 20 & Saudi Arabia & 339,267 & 5,043 & 325,330 & $34,963,754$ \\
\hline 21 & Indonesia & 336,716 & 11,935 & 258,519 & $274,329,617$ \\
\hline 22 & Turkey & 335,533 & 8,837 & 294,357 & $84,591,892$ \\
\hline 23 & Germany & 326,482 & 9,702 & 274,700 & $83,859,444$ \\
\hline 24 & Pakistan & 319,317 & 6,580 & 304,185 & $222,070,579$ \\
\hline 25 & Israel & 291,828 & 1,983 & 238,145 & $9,197,590$ \\
\hline 26 & Ukraine & 265,454 & 5,015 & 114,410 & $43,658,728$ \\
\hline 27 & Canada & 181,864 & 9,613 & 153,219 & $37,834,630$ \\
\hline 28 & Netherlands & 174,653 & 6,584 & N/A & $17,145,573$ \\
\hline 29 & Belgium & 162,258 & 10,191 & 20,272 & $11,603,814$ \\
\hline 30 & Romania & 155,283 & 5,411 & 117,942 & $19,201,008$ \\
\hline 31 & Morocco & 152,404 & 2,605 & 127,407 & $37,032,230$ \\
\hline 32 & Ecuador & 147,033 & 12,191 & 128,134 & $17,717,163$ \\
\hline 33 & Bolivia & 138,574 & 8,308 & 101,103 & $11,717,177$ \\
\hline 34 & Poland & 130,210 & 3,039 & 81,201 & $37,834,855$ \\
\hline 35 & Qatar & 127,985 & 220 & 124,978 & $2,807,805$ \\
\hline 36 & Panama & 120,313 & 2,491 & 96,164 & $4,333,539$ \\
\hline 37 & Dominican Republic & 118,477 & 2,173 & 94,084 & $10,878,250$ \\
\hline 38 & Czechia & 117,110 & 987 & 56,203 & $10,714,591$ \\
\hline 39 & Kuwait & 111,116 & 658 & 103,268 & $4,288,053$ \\
\hline 40 & Kazakhstan & 108,831 & 1,746 & 104,041 & $18,839,158$ \\
\hline 41 & Nepal & 107,755 & 636 & 75,804 & $29,281,110$ \\
\hline 42 & Oman & 106,575 & 1,046 & 93,222 & $5,141,941$ \\
\hline 43 & UAE & 106,229 & 445 & 97,284 & $9,923,623$ \\
\hline 44 & Egypt & 104,516 & 6,052 & 97,688 & $102,865,105$ \\
\hline 45 & Sweden & 98,451 & 5,894 & N/A & $10,116,932$ \\
\hline 46 & Guatemala & 97,715 & 3,384 & 86,582 & $18,006,746$ \\
\hline 47 & Japan & 88,912 & 1,627 & 81,824 & $126,366,347$ \\
\hline 48 & Costa Rica & 87,439 & 1,076 & 52,669 & $5,107,110$ \\
\hline 49 & Portugal & 86,664 & 2,080 & 53,187 & $10,188,255$ \\
\hline 50 & China & 85,578 & 4,634 & 80,714 & $1,439,323,776$ \\
\hline 51 & Ethiopia & 84,295 & 1,287 & 38,316 & $115,738,799$ \\
\hline 52 & Honduras & 84,081 & 2,512 & 32,012 & $9,948,132$ \\
\hline 53 & Belarus & 83,534 & 896 & 77,220 & $9,448,442$ \\
\hline 54 & Venezuela & 83,137 & 697 & 74,664 & $28,413,033$ \\
\hline 55 & Bahrain & 75,614 & 277 & 71,249 & $1,717,395$ \\
\hline 56 & Moldova & 62,151 & 1,461 & 44,357 & $4,031,299$ \\
\hline 57 & Uzbekistan & 61,205 & 507 & 58,069 & $33,603,532$ \\
\hline 58 & Switzerland & 60,368 & 2,088 & 48,400 & $8,672,349$ \\
\hline 59 & Nigeria & 60,266 & 1,115 & 51,735 & $207,530,415$ \\
\hline 60 & Singapore & 57,880 & 27 & 57,705 & $5,863,218$ \\
\hline
\end{tabular}


The Dynamics of Differential Impacts of COVID-19 on African Countries Compared to Other Parts of the World

\begin{tabular}{|c|c|c|c|c|c|}
\hline 61 & Armenia & 56,821 & 1,026 & 45,891 & $2,964,807$ \\
\hline 62 & Austria & 56,298 & 855 & 44,065 & $9,020,826$ \\
\hline 63 & Lebanon & 53,568 & 459 & 23,501 & $6,816,735$ \\
\hline 64 & Algeria & 53,072 & 1,801 & 37,170 & $44,069,055$ \\
\hline 65 & Paraguay & 49,675 & 1,077 & 32,090 & $7,156,887$ \\
\hline 66 & Kyrgyzstan & 49,528 & 1,090 & 44,522 & $6,553,907$ \\
\hline 67 & Ghana & 47,005 & 306 & 46,398 & $31,250,683$ \\
\hline 68 & Palestine & 44,299 & 381 & 37,942 & $5,133,781$ \\
\hline 69 & Libya & 42,712 & 631 & 24,038 & $6,897,205$ \\
\hline 70 & Ireland & 42,528 & 1,826 & 23,364 & $4,953,142$ \\
\hline 71 & Azerbaijan & 41,982 & 609 & 39,357 & $10,164,706$ \\
\hline 72 & Kenya & 41,546 & 766 & 32,000 & $54,095,241$ \\
\hline 73 & Afghanistan & 39,870 & 1,479 & 33,118 & $39,167,927$ \\
\hline 74 & Hungary & 38,837 & 968 & 11,037 & $9,653,380$ \\
\hline 75 & Serbia & 34,787 & 763 & 31,536 & $8,727,348$ \\
\hline 76 & Tunisia & 32,556 & 478 & 5,032 & $11,853,087$ \\
\hline 77 & Denmark & 32,422 & 669 & 26,380 & $5,797,948$ \\
\hline 78 & Bosnia and Herzegovina & 30,647 & 928 & 23,461 & $3,274,989$ \\
\hline 79 & El Salvador & 30,196 & 894 & 25,438 & $6,495,403$ \\
\hline 80 & Myanmar & 27,974 & 646 & 9,742 & $54,512,056$ \\
\hline 81 & Australia & 27,285 & 898 & 24,998 & $25,582,199$ \\
\hline 82 & Jordan & 24,926 & 191 & 6,101 & $10,231,389$ \\
\hline 83 & S. Korea & 24,703 & 433 & 22,729 & $51,281,663$ \\
\hline 84 & Bulgaria & 24,402 & 892 & 15,847 & $6,933,476$ \\
\hline 85 & Greece & 22,358 & 449 & 9,989 & $10,408,537$ \\
\hline 86 & Cameroon & 21,203 & 423 & 20,117 & $26,725,710$ \\
\hline 87 & North Macedonia & 20,937 & 792 & 16,194 & $2,083,350$ \\
\hline 88 & Croatia & 20,621 & 327 & 17,582 & $4,098,036$ \\
\hline 89 & Slovakia & 20,355 & 61 & 6,031 & $5,460,390$ \\
\hline 90 & Ivory Coast & 20,154 & 120 & 19,798 & $26,556,096$ \\
\hline 91 & Madagascar & 16,718 & 237 & 16,042 & $27,884,498$ \\
\hline 92 & Malaysia & 15,657 & 157 & 10,913 & $32,481,150$ \\
\hline 93 & Norway & 15,524 & 275 & 11,863 & $5,433,105$ \\
\hline 94 & Zambia & 15,458 & 337 & 14,599 & $18,523,331$ \\
\hline 95 & Albania & 15,399 & 420 & 9,500 & $2,876,906$ \\
\hline 96 & Senegal & 15,268 & 314 & 13,297 & $16,863,717$ \\
\hline 97 & Montenegro & 13,869 & 202 & 9,620 & 628,089 \\
\hline 98 & Sudan & 13,685 & 836 & 6,764 & $44,128,647$ \\
\hline 99 & Georgia & 12,272 & 92 & 6,538 & $3,986,993$ \\
\hline 100 & Finland & 11,998 & 346 & 8,500 & $5,543,152$ \\
\hline 101 & Namibia & 11,936 & 128 & 9,817 & $2,553,573$ \\
\hline 102 & Guinea & 11,022 & 69 & 10,324 & $13,229,375$ \\
\hline 103 & Maldives & 10,894 & 35 & 9,742 & 543,167 \\
\hline 104 & DRC & 10,851 & 276 & 10,242 & $90,295,401$ \\
\hline 105 & Tajikistan & 10,222 & 79 & 9,058 & $9,596,192$ \\
\hline 106 & French Guiana & 10,144 & 69 & 9,834 & 300,785 \\
\hline 107 & Mozambique & 10,001 & 71 & 7,338 & $31,492,481$ \\
\hline 108 & Uganda & 9,801 & 93 & 6,109 & $46,129,601$ \\
\hline 109 & Luxembourg & 9,722 & 132 & 8,038 & 628,790 \\
\hline 110 & Haiti & 8,882 & 230 & 7,104 & $11,441,165$ \\
\hline 111 & Gabon & 8,835 & 54 & 8,189 & $2,240,060$ \\
\hline 112 & Slovenia & 8,663 & 167 & 5,182 & $2,079,019$ \\
\hline 113 & Zimbabwe & 8,011 & 230 & 6,504 & $14,922,836$ \\
\hline 114 & Jamaica & 7,718 & 139 & 3,162 & $2,964,803$ \\
\hline 115 & Mauritania & 7,550 & 163 & 7,274 & $4,682,842$ \\
\hline 116 & Cabo Verde & 7,072 & 75 & 5,981 & 557,669 \\
\hline 117 & Guadeloupe & 6,483 & 77 & 2,199 & 400,143 \\
\hline
\end{tabular}


The Dynamics of Differential Impacts of COVID-19 on African Countries Compared to Other Parts of the World

\begin{tabular}{|c|c|c|c|c|c|}
\hline 118 & Angola & 6,366 & 218 & 2,743 & $33,141,527$ \\
\hline 119 & Lithuania & 6,248 & 103 & 2,793 & $2,711,324$ \\
\hline 120 & Cuba & 5,978 & 123 & 5,540 & $11,324,657$ \\
\hline 121 & Malawi & 5,821 & 180 & 4,647 & $19,264,273$ \\
\hline 122 & Congo & 5,118 & 90 & 3,887 & $5,555,071$ \\
\hline 123 & Trinidad and Tobago & 5,101 & 90 & 3,252 & $1,400,765$ \\
\hline 124 & Bahamas & 5,078 & 107 & 2,900 & 394,293 \\
\hline 125 & Equatorial Guinea & 5,063 & 83 & 4,894 & $1,415,356$ \\
\hline 126 & Rwanda & 4,896 & 31 & 3,606 & $13,039,619$ \\
\hline 127 & CAR & 4,854 & 62 & 1,924 & $4,852,912$ \\
\hline 128 & Sri Lanka & 4,791 & 13 & 3,317 & $21,438,515$ \\
\hline 129 & Syria & 4,718 & 224 & 1,296 & $17,616,485$ \\
\hline 130 & Réunion & 4,491 & 16 & 3,994 & 897,102 \\
\hline 131 & Aruba & 4,194 & 33 & 3,768 & 106,894 \\
\hline 132 & Mayotte & 3,989 & 43 & 2,964 & 274,609 \\
\hline 133 & Estonia & 3,883 & 68 & 2,967 & $1,326,787$ \\
\hline 134 & Somalia & 3,864 & 99 & 3,089 & $16,013,278$ \\
\hline 135 & Malta & 3,776 & 41 & 2,967 & 441,875 \\
\hline 136 & Thailand & 3,641 & 59 & 3,454 & $69,849,390$ \\
\hline 137 & Gambia & 3,632 & 117 & 2,543 & $2,435,044$ \\
\hline 138 & Iceland & 3,526 & 10 & 2,499 & 341,864 \\
\hline 139 & Guyana & 3,469 & 103 & 2,318 & 787,619 \\
\hline 140 & Mali & 3,286 & 132 & 2,527 & $20,408,512$ \\
\hline 141 & Botswana & 3,219 & 18 & 834 & $2,364,654$ \\
\hline 142 & South Sudan & 2,777 & 55 & 1,290 & $11,230,230$ \\
\hline 143 & Latvia & 2,765 & 40 & 1,325 & $1,880,201$ \\
\hline 144 & Benin & 2,411 & 41 & 1,973 & $12,209,431$ \\
\hline 145 & Guinea-Bissau & 2,385 & 40 & 1,728 & $1,980,688$ \\
\hline 146 & Sierra Leone & 2,306 & 72 & 1,736 & $8,021,477$ \\
\hline 147 & Uruguay & 2,294 & 50 & 1,942 & $3,477,121$ \\
\hline 148 & Burkina Faso & 2,280 & 63 & 1,554 & $21,058,597$ \\
\hline 149 & Yemen & 2,052 & 595 & 1,329 & $30,005,627$ \\
\hline 150 & Togo & 1,940 & 49 & 1,457 & $8,331,665$ \\
\hline 151 & New Zealand & 1,871 & 25 & 1,801 & $5,002,100$ \\
\hline 152 & Martinique & 1,851 & 22 & 98 & 375,183 \\
\hline 153 & Lesotho & 1,805 & 42 & 961 & $2,147,001$ \\
\hline 154 & Liberia & 1,363 & 82 & 1,245 & $5,090,112$ \\
\hline 155 & Chad & 1,304 & 92 & 1,115 & $16,553,305$ \\
\hline 156 & Niger & 1,201 & 69 & 1,123 & $24,440,409$ \\
\hline 157 & Vietnam & 1,109 & 35 & 1,024 & $97,583,242$ \\
\hline 158 & Sao Tome and Principe & 929 & 15 & 892 & 220,279 \\
\hline 159 & Curaçao & 583 & 1 & 315 & 164,282 \\
\hline 160 & Papua New Guinea & 554 & 7 & 532 & $8,993,618$ \\
\hline 161 & Taiwan & 529 & 7 & 489 & $23,828,949$ \\
\hline 162 & Burundi & 524 & 1 & 472 & $11,986,354$ \\
\hline 163 & Tanzania & 509 & 21 & 183 & $60,194,425$ \\
\hline 164 & Comoros & 495 & 7 & 475 & 874,674 \\
\hline 165 & Gibraltar & 485 & & 413 & 33,688 \\
\hline 166 & Faeroe Islands & 477 & & 461 & 48,915 \\
\hline 167 & Saint Martin & 466 & 8 & 309 & 38,848 \\
\hline 168 & Eritrea & 414 & & 372 & $3,560,028$ \\
\hline 169 & Mauritius & 395 & 10 & 358 & $1,272,364$ \\
\hline 170 & Cambodia & 283 & & 278 & $16,783,110$ \\
\hline 171 & Monaco & 234 & 2 & 209 & 39,320 \\
\hline 172 & Cayman Islands & 221 & 1 & 211 & 65,937 \\
\hline 173 & Barbados & 208 & 7 & 186 & 287,474 \\
\hline 174 & Bermuda & 184 & 9 & 170 & 62,213 \\
\hline
\end{tabular}


The Dynamics of Differential Impacts of COVID-19 on African Countries Compared to Other Parts of the World

\begin{tabular}{|l|l|l|l|l|l|}
\hline 175 & Caribbean Netherlands & $\mathbf{1 4 8}$ & $\mathbf{2}$ & $\mathbf{7 8}$ & 26,291 \\
\hline 176 & Seychelles & $\mathbf{1 4 8}$ & & $\mathbf{1 4 4}$ & 98,518 \\
\hline 177 & Brunei & $\mathbf{1 4 6}$ & $\mathbf{3}$ & $\mathbf{1 4 3}$ & 438,648 \\
\hline 178 & Liechtenstein & $\mathbf{1 4 4}$ & $\mathbf{1}$ & $\mathbf{1 2 1}$ & 38,159 \\
\hline 179 & Antigua and Barbuda & $\mathbf{1 1 1}$ & $\mathbf{3}$ & $\mathbf{9 7}$ & 98,156 \\
\hline 180 & British Virgin Islands & $\mathbf{7 1}$ & $\mathbf{1}$ & $\mathbf{7 0}$ & 30,287 \\
\hline 181 & St. Barth & $\mathbf{6 5}$ & & $\mathbf{3 7}$ & 9,885 \\
\hline 182 & St. Vincent Grenadines & $\mathbf{6 4}$ & & $\mathbf{6 4}$ & 111,039 \\
\hline 183 & Macao & $\mathbf{4 6}$ & & $\mathbf{4 6}$ & 651,790 \\
\hline 184 & Fiji & $\mathbf{3 2}$ & $\mathbf{2}$ & $\mathbf{2 8}$ & 898,265 \\
\hline 185 & Dominica & $\mathbf{3 2}$ & & $\mathbf{2 4}$ & 72,036 \\
\hline 186 & Saint Lucia & $\mathbf{2 9}$ & & $\mathbf{2 7}$ & 183,863 \\
\hline 187 & Timor-Leste & $\mathbf{2 9}$ & & $\mathbf{2 8}$ & $1,325,347$ \\
\hline
\end{tabular}

Sources and data used were provided under Latest Updates from WHO/World meter's on October 12, 2020

Infectious, recovery and mortality rate of COVID-19 based on country and relative to the USA

When compared against USA, African countries were among the least infected, more recovery and lesser number of deaths.

Table 2: Infectious, recovery and mortality rate of COVID-19 based on country and relative to the USA

\begin{tabular}{|c|c|c|c|c|c|}
\hline & Country, & $\%$ of $A$ to $D$ & $\%$ of $B$ to $D$ & $E / 2.41$ & $F / 0.066$ \\
\hline $\mathrm{S} / \mathrm{N}$ & Other & (E) & (G) & $\left(F_{1}\right)$ & $\left(F_{2}\right)$ \\
\hline 1 & USA & 2.411 & 0.066 & 1.00 & 1.00 \\
\hline 2 & India & 0.515 & 0.008 & 0.21 & 0.12 \\
\hline 3 & Brazil & 2.392 & 0.071 & 0.99 & 1.07 \\
\hline 4 & Russia & 0.899 & 0.016 & 0.37 & 0.24 \\
\hline 5 & Colombia & 1.786 & 0.055 & 0.74 & 0.83 \\
\hline 6 & Argentina & 1.973 & 0.053 & 0.82 & 0.80 \\
\hline 7 & Spain & 1.904 & 0.070 & 0.79 & 1.07 \\
\hline 8 & Peru & 2.566 & 0.101 & 1.06 & 1.52 \\
\hline 9 & Mexico & 0.632 & 0.065 & 0.26 & 0.98 \\
\hline 10 & France & 1.125 & 0.050 & 0.47 & 0.76 \\
\hline 11 & South Africa & 1.163 & 0.030 & 0.48 & 0.45 \\
\hline 12 & UK & 0.888 & 0.063 & 0.37 & 0.95 \\
\hline 13 & Iran & 0.593 & 0.034 & 0.25 & 0.51 \\
\hline 14 & Chile & 2.512 & 0.070 & 1.04 & 1.05 \\
\hline 15 & Iraq & 0.994 & 0.024 & 0.41 & 0.37 \\
\hline 16 & Bangladesh & 0.230 & 0.003 & 0.10 & 0.05 \\
\hline 17 & Italy & 0.587 & 0.060 & 0.24 & 0.91 \\
\hline 18 & Philippines & 0.312 & 0.006 & 0.13 & 0.09 \\
\hline 19 & Saudi Arabia & 0.970 & 0.014 & 0.40 & 0.22 \\
\hline 20 & Indonesia & 0.123 & 0.004 & 0.05 & 0.07 \\
\hline 21 & Turkey & 0.397 & 0.010 & 0.16 & 0.16 \\
\hline 22 & Germany & 0.389 & 0.012 & 0.16 & 0.18 \\
\hline 23 & Pakistan & 0.144 & 0.003 & 0.06 & 0.04 \\
\hline 24 & Israel & 3.173 & 0.022 & 1.32 & 0.33 \\
\hline 25 & Ukraine & 0.608 & 0.011 & 0.25 & 0.17 \\
\hline 26 & Canada & 0.481 & 0.025 & 0.20 & 0.38 \\
\hline 27 & Netherlands & 1.019 & 0.038 & 0.42 & 0.58 \\
\hline 28 & Belgium & 1.398 & 0.088 & 0.58 & 1.33 \\
\hline 29 & Romania & 0.809 & 0.028 & 0.34 & 0.43 \\
\hline 30 & Morocco & 0.412 & 0.007 & 0.17 & 0.11 \\
\hline 31 & Ecuador & 0.830 & 0.069 & 0.34 & 1.04 \\
\hline
\end{tabular}


The Dynamics of Differential Impacts of COVID-19 on African Countries Compared to Other Parts of the World

\begin{tabular}{|c|c|c|c|c|c|}
\hline 32 & Bolivia & 1.183 & 0.071 & 0.49 & 1.07 \\
\hline 33 & Poland & 0.344 & 0.008 & 0.14 & 0.12 \\
\hline 34 & Qatar & 4.558 & 0.008 & 1.89 & 0.12 \\
\hline 35 & Panama & 2.776 & 0.057 & 1.15 & 0.87 \\
\hline 36 & Dominican Republic & 1.089 & 0.020 & 0.45 & 0.30 \\
\hline 37 & Czechia & 1.093 & 0.009 & 0.45 & 0.14 \\
\hline 38 & Kuwait & 2.591 & 0.015 & 1.08 & 0.23 \\
\hline 39 & Kazakhstan & 0.578 & 0.009 & 0.24 & 0.14 \\
\hline 40 & Nepal & 0.368 & 0.002 & 0.15 & 0.03 \\
\hline 41 & Oman & 2.073 & 0.020 & 0.86 & 0.31 \\
\hline 42 & UAE & 1.070 & 0.004 & 0.44 & 0.07 \\
\hline 43 & Egypt & 0.102 & 0.006 & 0.04 & 0.09 \\
\hline 44 & Sweden & 0.973 & 0.058 & 0.40 & 0.88 \\
\hline 45 & Guatemala & 0.543 & 0.019 & 0.23 & 0.28 \\
\hline 46 & Japan & 0.070 & 0.001 & 0.03 & 0.02 \\
\hline 47 & Costa Rica & 1.712 & 0.021 & 0.71 & 0.32 \\
\hline 48 & Portugal & 0.851 & 0.020 & 0.35 & 0.31 \\
\hline 49 & China & 0.006 & 0.000 & 0.00 & 0.00 \\
\hline 50 & Ethiopia & 0.073 & 0.001 & 0.03 & 0.02 \\
\hline 51 & Honduras & 0.845 & 0.025 & 0.35 & 0.38 \\
\hline 52 & Belarus & 0.884 & 0.009 & 0.37 & 0.14 \\
\hline 53 & Venezuela & 0.293 & 0.002 & 0.12 & 0.04 \\
\hline 54 & Bahrain & 4.403 & 0.016 & 1.83 & 0.24 \\
\hline 55 & Moldova & 1.542 & 0.036 & 0.64 & 0.55 \\
\hline 56 & Uzbekistan & 0.182 & 0.002 & 0.08 & 0.02 \\
\hline 57 & Switzerland & 0.696 & 0.024 & 0.29 & 0.36 \\
\hline 58 & Nigeria & 0.029 & 0.001 & 0.01 & 0.01 \\
\hline 59 & Singapore & 0.987 & 0.000 & 0.41 & 0.01 \\
\hline 60 & Armenia & 1.917 & 0.035 & 0.80 & 0.52 \\
\hline 61 & Austria & 0.624 & 0.009 & 0.26 & 0.14 \\
\hline 62 & Lebanon & 0.786 & 0.007 & 0.33 & 0.10 \\
\hline 63 & Algeria & 0.120 & 0.004 & 0.05 & 0.06 \\
\hline 64 & Paraguay & 0.694 & 0.015 & 0.29 & 0.23 \\
\hline 65 & Kyrgyzstan & 0.756 & 0.017 & 0.31 & 0.25 \\
\hline 66 & Ghana & 0.150 & 0.001 & 0.06 & 0.01 \\
\hline 67 & Palestine & 0.863 & 0.007 & 0.36 & 0.11 \\
\hline 68 & Libya & 0.619 & 0.009 & 0.26 & 0.14 \\
\hline 69 & Ireland & 0.859 & 0.037 & 0.36 & 0.56 \\
\hline 70 & Azerbaijan & 0.413 & 0.006 & 0.17 & 0.09 \\
\hline 71 & Kenya & 0.077 & 0.001 & 0.03 & 0.02 \\
\hline 72 & Afghanistan & 0.102 & 0.004 & 0.04 & 0.06 \\
\hline 73 & Hungary & 0.402 & 0.010 & 0.17 & 0.15 \\
\hline 74 & Serbia & 0.399 & 0.009 & 0.17 & 0.13 \\
\hline 75 & Tunisia & 0.275 & 0.004 & 0.11 & 0.06 \\
\hline 76 & Denmark & 0.559 & 0.012 & 0.23 & 0.17 \\
\hline 77 & Bosnia and Herzegovina & 0.936 & 0.028 & 0.39 & 0.43 \\
\hline 78 & El Salvador & 0.465 & 0.014 & 0.19 & 0.21 \\
\hline 79 & Myanmar & 0.051 & 0.001 & 0.02 & 0.02 \\
\hline 80 & Australia & 0.107 & 0.004 & 0.04 & 0.05 \\
\hline 81 & Jordan & 0.244 & 0.002 & 0.10 & 0.03 \\
\hline
\end{tabular}


The Dynamics of Differential Impacts of COVID-19 on African Countries Compared to Other Parts of the World

\begin{tabular}{|c|c|c|c|c|c|}
\hline 82 & S. Korea & 0.048 & 0.001 & 0.02 & 0.01 \\
\hline 83 & Bulgaria & 0.352 & 0.013 & 0.15 & 0.19 \\
\hline 84 & Greece & 0.215 & 0.004 & 0.09 & 0.07 \\
\hline 85 & Cameroon & 0.079 & 0.002 & 0.03 & 0.02 \\
\hline 86 & North Macedonia & 1.005 & 0.038 & 0.42 & 0.58 \\
\hline 87 & Croatia & 0.503 & 0.008 & 0.21 & 0.12 \\
\hline 88 & Slovakia & 0.373 & 0.001 & 0.15 & 0.02 \\
\hline 89 & Ivory Coast & 0.076 & 0.000 & 0.03 & 0.01 \\
\hline 90 & Madagascar & 0.060 & 0.001 & 0.02 & 0.01 \\
\hline 91 & Malaysia & 0.048 & 0.000 & 0.02 & 0.01 \\
\hline 92 & Norway & 0.286 & 0.005 & 0.12 & 0.08 \\
\hline 93 & Zambia & 0.083 & 0.002 & 0.03 & 0.03 \\
\hline 94 & Albania & 0.535 & 0.015 & 0.22 & 0.22 \\
\hline 95 & Senegal & 0.091 & 0.002 & 0.04 & 0.03 \\
\hline 96 & Montenegro & 2.208 & 0.032 & 0.92 & 0.49 \\
\hline 97 & Sudan & 0.031 & 0.002 & 0.01 & 0.03 \\
\hline 98 & Georgia & 0.308 & 0.002 & 0.13 & 0.03 \\
\hline 99 & Finland & 0.216 & 0.006 & 0.09 & 0.09 \\
\hline 100 & Namibia & 0.467 & 0.005 & 0.19 & 0.08 \\
\hline 101 & Guinea & 0.083 & 0.001 & 0.03 & 0.01 \\
\hline 102 & Maldives & 2.006 & 0.006 & 0.83 & 0.10 \\
\hline 103 & DRC & 0.012 & 0.000 & 0.00 & 0.00 \\
\hline 104 & Tajikistan & 0.107 & 0.001 & 0.04 & 0.01 \\
\hline 105 & French Guiana & 3.373 & 0.023 & 1.40 & 0.35 \\
\hline 106 & Mozambique & 0.032 & 0.000 & 0.01 & 0.00 \\
\hline 107 & Uganda & 0.021 & 0.000 & 0.01 & 0.00 \\
\hline 108 & Luxembourg & 1.546 & 0.021 & 0.64 & 0.32 \\
\hline 109 & Haiti & 0.078 & 0.002 & 0.03 & 0.03 \\
\hline 110 & Gabon & 0.394 & 0.002 & 0.16 & 0.04 \\
\hline 111 & Slovenia & 0.417 & 0.008 & 0.17 & 0.12 \\
\hline 112 & Zimbabwe & 0.054 & 0.002 & 0.02 & 0.02 \\
\hline 113 & Jamaica & 0.260 & 0.005 & 0.11 & 0.07 \\
\hline 114 & Mauritania & 0.161 & 0.003 & 0.07 & 0.05 \\
\hline 115 & Cabo Verde & 1.268 & 0.013 & 0.53 & 0.20 \\
\hline 116 & Guadeloupe & 1.620 & 0.019 & 0.67 & 0.29 \\
\hline 117 & Angola & 0.019 & 0.001 & 0.01 & 0.01 \\
\hline 118 & Lithuania & 0.230 & 0.004 & 0.10 & 0.06 \\
\hline 119 & Cuba & 0.053 & 0.001 & 0.02 & 0.02 \\
\hline 120 & Malawi & 0.030 & 0.001 & 0.01 & 0.01 \\
\hline 121 & Congo & 0.092 & 0.002 & 0.04 & 0.02 \\
\hline 122 & Trinidad and Tobago & 0.364 & 0.006 & 0.15 & 0.10 \\
\hline 123 & Bahamas & 1.288 & 0.027 & 0.53 & 0.41 \\
\hline 124 & Equatorial Guinea & 0.358 & 0.006 & 0.15 & 0.09 \\
\hline 125 & Rwanda & 0.038 & 0.000 & 0.02 & 0.00 \\
\hline 126 & CAR & 0.100 & 0.001 & 0.04 & 0.02 \\
\hline 127 & Sri Lanka & 0.022 & 0.000 & 0.01 & 0.00 \\
\hline 128 & Syria & 0.027 & 0.001 & 0.01 & 0.02 \\
\hline 129 & Réunion & 0.501 & 0.002 & 0.21 & 0.03 \\
\hline 130 & Aruba & 3.924 & 0.031 & 1.63 & 0.47 \\
\hline 131 & Mayotte & 1.453 & 0.016 & 0.60 & 0.24 \\
\hline
\end{tabular}


The Dynamics of Differential Impacts of COVID-19 on African Countries Compared to Other Parts of the World

\begin{tabular}{|c|c|c|c|c|c|}
\hline 132 & Estonia & 0.293 & 0.005 & 0.12 & 0.08 \\
\hline 133 & Somalia & 0.024 & 0.001 & 0.01 & 0.01 \\
\hline 134 & Malta & 0.855 & 0.009 & 0.35 & 0.14 \\
\hline 135 & Thailand & 0.005 & 0.000 & 0.00 & 0.00 \\
\hline 136 & Gambia & 0.149 & 0.005 & 0.06 & 0.07 \\
\hline 137 & Iceland & 1.031 & 0.003 & 0.43 & 0.04 \\
\hline 138 & Guyana & 0.440 & 0.013 & 0.18 & 0.20 \\
\hline 139 & Mali & 0.016 & 0.001 & 0.01 & 0.01 \\
\hline 140 & Botswana & 0.136 & 0.001 & 0.06 & 0.01 \\
\hline 141 & South Sudan & 0.025 & 0.000 & 0.01 & 0.01 \\
\hline 142 & Latvia & 0.147 & 0.002 & 0.06 & 0.03 \\
\hline 143 & Benin & 0.020 & 0.000 & 0.01 & 0.01 \\
\hline 144 & Guinea-Bissau & 0.120 & 0.002 & 0.05 & 0.03 \\
\hline 145 & Sierra Leone & 0.029 & 0.001 & 0.01 & 0.01 \\
\hline 146 & Uruguay & 0.066 & 0.001 & 0.03 & 0.02 \\
\hline 147 & Burkina Faso & 0.011 & 0.000 & 0.00 & 0.00 \\
\hline 148 & Yemen & 0.007 & 0.002 & 0.00 & 0.03 \\
\hline 149 & Togo & 0.023 & 0.001 & 0.01 & 0.01 \\
\hline 150 & New Zealand & 0.037 & 0.000 & 0.02 & 0.01 \\
\hline 151 & Martinique & 0.493 & 0.006 & 0.20 & 0.09 \\
\hline 152 & Lesotho & 0.084 & 0.002 & 0.03 & 0.03 \\
\hline 153 & Liberia & 0.027 & 0.002 & 0.01 & 0.02 \\
\hline 154 & Chad & 0.008 & 0.001 & 0.00 & 0.01 \\
\hline 155 & Niger & 0.005 & 0.000 & 0.00 & 0.00 \\
\hline 156 & Vietnam & 0.001 & 0.000 & 0.00 & 0.00 \\
\hline 157 & Sao Tome and Principe & 0.422 & 0.007 & 0.17 & 0.10 \\
\hline 158 & Curaçao & 0.355 & 0.001 & 0.15 & 0.01 \\
\hline 159 & Papua New Guinea & 0.006 & 0.000 & 0.00 & 0.00 \\
\hline 160 & Taiwan & 0.002 & 0.000 & 0.00 & 0.00 \\
\hline 161 & Burundi & 0.004 & 0.000 & 0.00 & 0.00 \\
\hline 162 & Tanzania & 0.001 & 0.000 & 0.00 & 0.00 \\
\hline 163 & Comoros & 0.057 & 0.001 & 0.02 & 0.01 \\
\hline 164 & Gibraltar & 1.440 & 0.000 & 0.60 & 0.00 \\
\hline 165 & Faeroe Islands & 0.975 & 0.000 & 0.40 & 0.00 \\
\hline 166 & Saint Martin & 1.200 & 0.021 & 0.50 & 0.31 \\
\hline 167 & Eritrea & 0.012 & 0.000 & 0.00 & 0.00 \\
\hline 168 & Mauritius & 0.031 & 0.001 & 0.01 & 0.01 \\
\hline 169 & Cambodia & 0.002 & 0.000 & 0.00 & 0.00 \\
\hline 170 & Monaco & 0.595 & 0.005 & 0.25 & 0.08 \\
\hline 171 & Cayman Islands & 0.335 & 0.002 & 0.14 & 0.02 \\
\hline 172 & Barbados & 0.072 & 0.002 & 0.03 & 0.04 \\
\hline 173 & Bermuda & 0.296 & 0.014 & 0.12 & 0.22 \\
\hline 174 & Caribbean Netherlands & 0.563 & 0.008 & 0.23 & 0.12 \\
\hline 175 & Seychelles & 0.150 & 0.000 & 0.06 & 0.00 \\
\hline 176 & Brunei & 0.033 & 0.001 & 0.01 & 0.01 \\
\hline 177 & Liechtenstein & 0.377 & 0.003 & 0.16 & 0.04 \\
\hline 178 & Antigua and Barbuda & 0.113 & 0.003 & 0.05 & 0.05 \\
\hline 179 & British Virgin Islands & 0.234 & 0.003 & 0.10 & 0.05 \\
\hline 180 & St. Barth & 0.658 & 0.000 & 0.27 & 0.00 \\
\hline 181 & St. Vincent Grenadines & 0.058 & 0.000 & 0.02 & 0.00 \\
\hline
\end{tabular}


The Dynamics of Differential Impacts of COVID-19 on African Countries Compared to Other Parts of the World

\begin{tabular}{|l|l|l|l|l|l|}
\hline 182 & Macao & 0.007 & 0.000 & 0.00 & 0.00 \\
\hline 183 & Fiji & 0.004 & 0.000 & 0.00 & 0.00 \\
\hline 184 & Dominica & 0.044 & 0.000 & 0.02 & 0.00 \\
\hline 185 & Saint Lucia & 0.016 & 0.000 & 0.01 & 0.00 \\
\hline 186 & Timor-Leste & 0.002 & 0.000 & 0.00 & 0.00 \\
\hline
\end{tabular}

Figures obtained for USA were used as the comparism factor (CF), which is a ratio of figure obtained to the respective country population divided by the value obtained for USA.

Values of $F_{1}$ and $F_{2}$ represent case/incidence and mortality index.

Factor of more than 1 = very high infection and mortality index

Factor of approximately $1=$ high infection and mortality index

Factor of $\leq 1$ but $\geq 0.5$ = moderately high infection and mortality index

Factor of $\leq 0.5$ but $\geq 0.1$ = low infection and mortality index

Factor of $<0.1$ = very low infection, mortality and recovery index

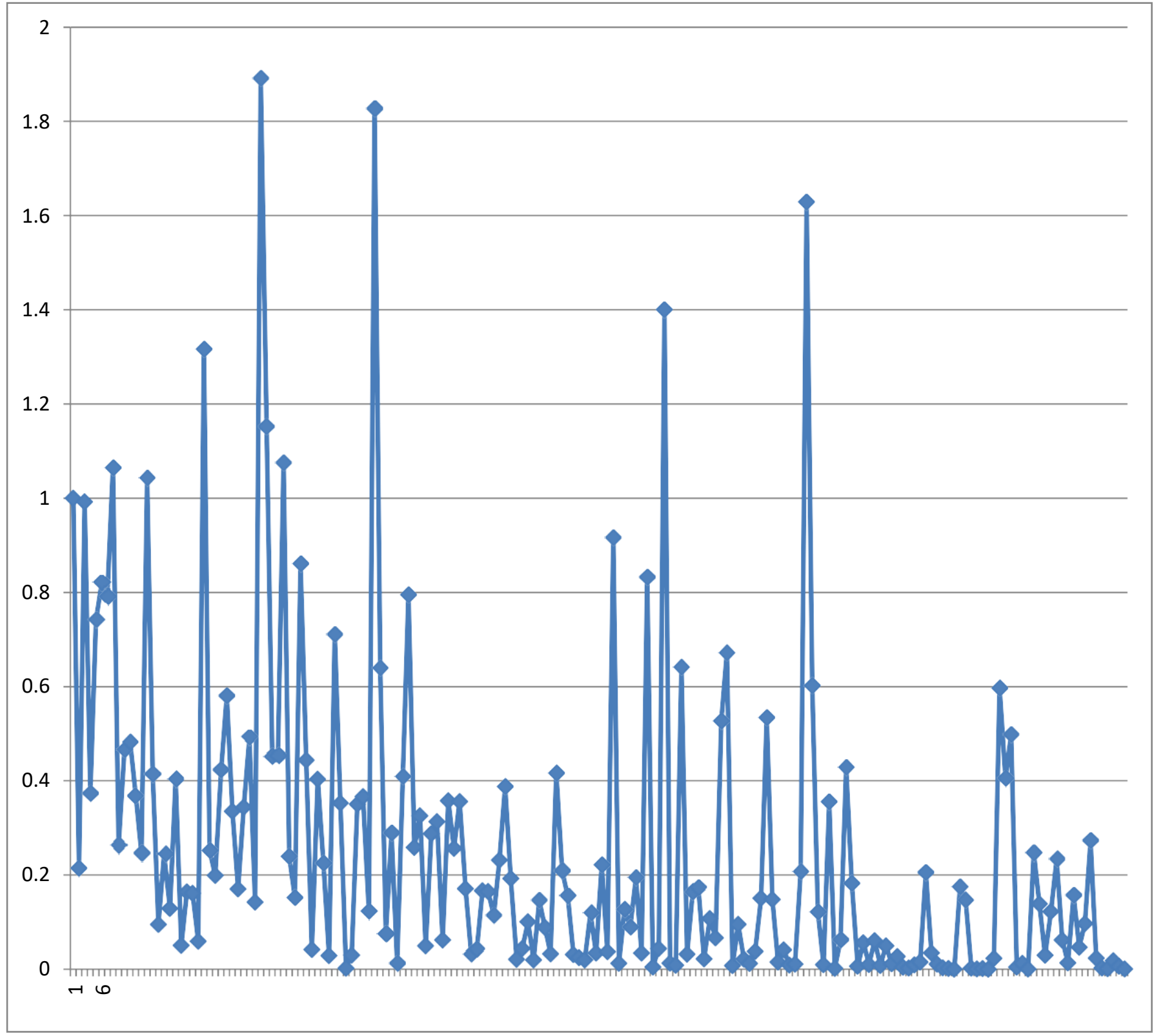

Figure 1: graph comparing infection per country relative to USA 


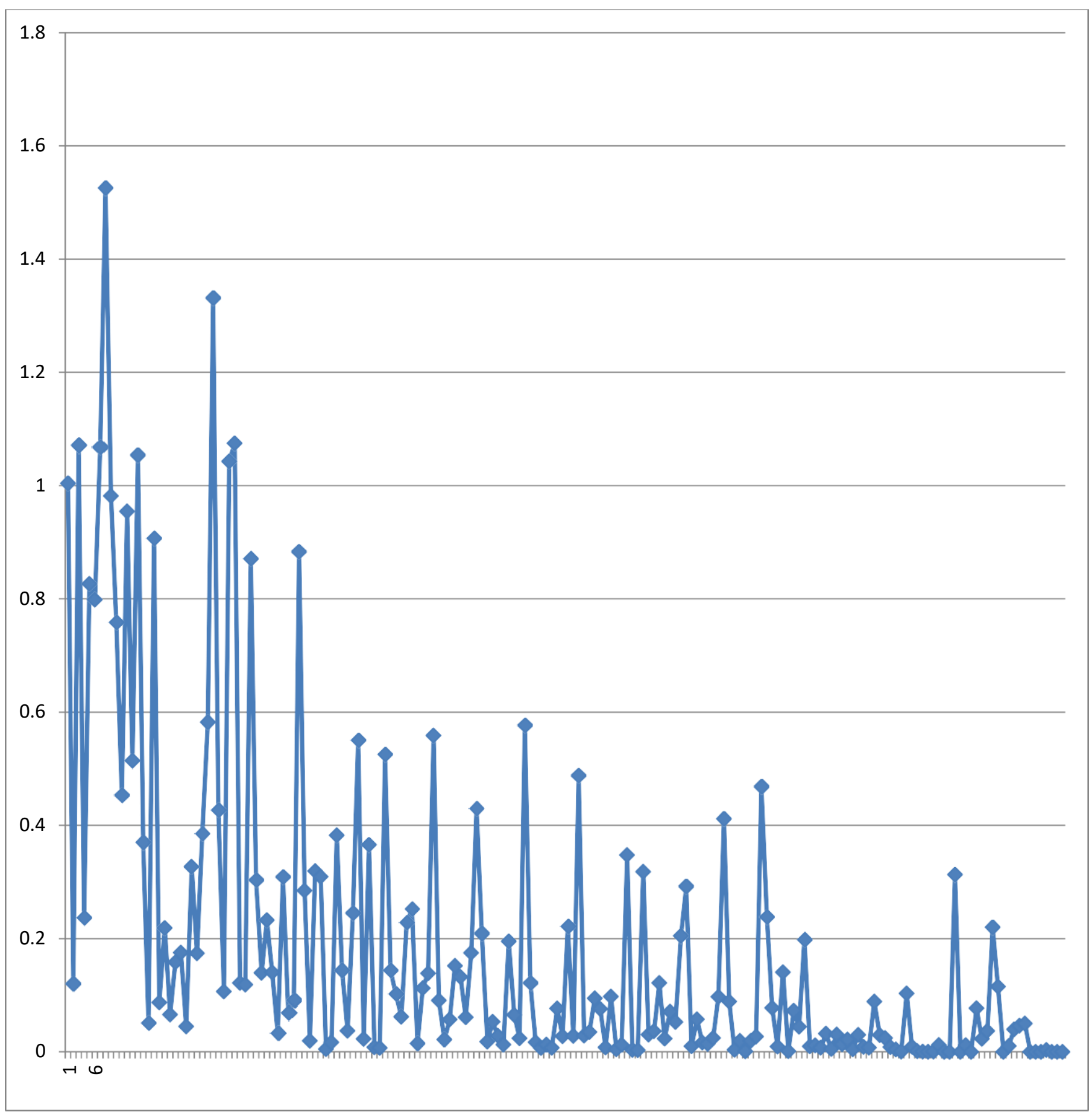

Figure 2: graph comparing death per country relative to USA

\section{DISCUSSION}

Africa is the world's second-largest and second-most populous continent, after Asia ${ }^{23,24,25,26}$. It is confronted by a heavy burden of communicable and non-communicable diseases ${ }^{23,25}$. Cost-effective interventions that can prevent the disease burden exist but coverage is too low due to health systems weaknesses ${ }^{27,28}$. The World Health Organization (WHO) has rushed to beef up the ability of African countries to test for the virus and train health professionals in caring for people affected by it ${ }^{2}$. From the table above South Africa is the only African country with moderately high infection and mortality. Morocco, Ghana, Equatorial Guinea etc. have low infection and mortality, while most African countries have very low infection and mortality. Most countries in Africa have a communal lifestyle compared to the isolation lifestyle of the western countries. Hence, it is possible that a large proportion of Africans may have been exposed to COVID-19 but few have presented visible symptoms or tested positive to the virus. Interestingly, Africa-American are the most hit among other Americans by this pandemic ${ }^{29}$.

It is expected that due to the virus mode of transmission, the index value is supposed to be very high for African countries. There have been several suggestions to explain why Africa appears to be less affected by the pandemic. These include notable 


\section{The Dynamics of Differential Impacts of COVID-19 on African Countries Compared to Other Parts of the World}

difference in the population age pyramids of USA, European and African countries, slow or low testing rate, low records of hospital reporting the disease, relatively high temperature and high humidity and high consumption of antioxidant food/fruit substances $^{25}$. Because of the density of the virus in tropical Africa, it cannot travel far. Hence, the recommendation of two meters of social distancing rule does not apply because the virus cannot go far. Africans food contents also raised the diet factor. African foods have a lot of zinc, vitamin C and other antioxidants. They take all these on a daily basis without even thinking about it $^{25}$. They also take a lot of phyto medicines which contains a lot of antioxidant ${ }^{30}$.

Evolutionary or adaptive immune response to the virus may also have immensely contributed to low infectious and mortality case in Africa. The African-American population is more affected by COVID-19 when compared to other Americans in the $\mathrm{USA}^{30,31,32,33}$. Therefore, it is unlikely to be genetic, but more likely related to the environment. A study showed that children in low- and middle-income countries experience a high incidence of infectious disease in their first years of life ${ }^{34,35,36,37}$. Babraham Institute (2020) studied immune responses of African children compared to Dutch children. They found that the immune systems of African children develop faster than those of Dutch children ${ }^{38}$. Exposure to germs in childhood may have helped to strengthen the immune system and protect children from developing allergies, asthma and other infectious diseases, on subsequence exposure to the same/similar allergen/pathogen or cross allergen/pathogen. This supports the 'hygiene hypothesis ${ }^{39,40,41,42}$, which contends that such diseases are more common in the developed world where the prevalence of antibiotics and antibacterial reduce children's exposure to microbes ${ }^{43,44,45}$. Thus, early exposure to some diseases in Africa may have resulted in a more robust innate and/or adaptive immune response. As a result countries in Africa are both vulnerable and potentially more resilient to the corona virus.

The immune system is a host defense system comprising many biological structures and processes within an organism that protects against disease ${ }^{46}$. To function properly, an immune system must detect a wide variety of agents, known as pathogens, from viruses to parasitic worms, and distinguish them from the organism's own healthy tissue. The immune system protects its host from infection with layered defenses of increasing specificity. In simple terms, physical barriers prevent pathogens such as bacteria and viruses from entering the organism ${ }^{47}$. If a pathogen breaches these barriers, the innate immune system provides an immediate, but non-specific response. Innate immune systems are found in all plants and animals. The innate response is usually triggered when microbes are identified by pattern recognition receptors, which recognize components that are conserved among broad groups of microorganisms, ${ }^{48}$ or when damaged, injured or stressed cells send out alarm signals, many of which (but not all) are recognized by the same receptors as those that recognize pathogens. ${ }^{49}$ Innate immune defenses are nonspecific, meaning these systems respond to pathogens in a generic way. ${ }^{50}$ This system does not confer longlasting immunity against a pathogen. ${ }^{50}$

If pathogens successfully evade the innate response, vertebrates possess a second layer of protection, the adaptive immune system, which is activated by the innate response. Here, the immune system adapts its response during an infection to improve its recognition of the pathogen. This improved response is then retained after the pathogen has been eliminated, in the form of an immunological memory, and allows the adaptive immune system to mount faster and stronger attacks each time this pathogen is encountered. The adaptive immune system evolved in early vertebrates and allows for a stronger immune response as well as immunological memory, where each pathogen is "remembered" by a signature antigen. ${ }^{50}$ The adaptive immune response is antigen-specific and requires the recognition of specific non-self antigens during a process called antigen presentation. Antigen specificity allows for the generation of responses that are tailored to specific pathogens or pathogeninfected cells. The ability to mount these tailored responses is maintained in the body by memory cells. ${ }^{47,50}$

\section{CONCLUSION}

This is a unique study because the virus is new in many aspects. The study was focused on effect of COVID-19 on countries in Africa. African countries maybe more exposed to the virus, but they do not appear to be significantly affected by it. Many factors may have contributed to low case and mortality, such as the population age pyramids, relatively high temperature and high humidity, high consumption of antioxidant food/fruit substances, evolutionary and/or adaptive immune response. Also, Africa is home to several viral diseases such as dengue fever, small pox, chicken pox, measles, Ebola and polio disease some of which the body system has developed and evolved a way to cope with. This may have resulted in direct or indirect cross immune response/defense that is beneficial on exposure to same, similar or different viral infection including corona virus disease.

Also, the communal system and poor government health regulation in Africa would have allowed for rapid transmission of the virus from person(s) to person(s) within the shortest possible time. This means most Africans may have been exposed to the virus without showing noticeable symptoms and may have recovered, but very few people have shown symptom to it. Therefore, there is need for COVID-19 antibody testing, which will reveal the true picture of who has been exposed than the current antigen testing which only provides active disease information. 


\section{The Dynamics of Differential Impacts of COVID-19 on African Countries Compared to Other Parts of the World}

The study also shows that Africa need vaccine, but vaccination may relatively not be an emergency when compared to western world because most individuals in Africa countries may have been naturally and unconsciously inoculated.

\section{Recommendation}

More studies and surveys need to be conducted to understand why and how primarily the virus affects Africa and it significances to Africa and maybe the world.

\section{CONFLICT OF INTEREST}

The authors declare that there are not any potential conflicts of interest.

\section{ACKNOWLEDGEMENT}

The authors wish to appreciate and thank everyone who has contributed to the success of this study. Special appreciation to United Nations Geo scheme and WHO for access to raw data per country was gotten.

\section{REFERENCE}

1) Virus Taxonomy. (2020) Release. International Committee on Taxonomy of Viruses (ICTV). March 2019. Archived from the original on 2018-03-04. Retrieved 2020-01-24

2) www.who.int/emergencies/diseases/novel-coronavirus-2020

3) Virus Taxonomy: (2019) Release". International Committee on Taxonomy of Viruses (ICTV).

4) Giaimo C (2020). The Spiky Blob Seen Around the World. The New York Times. Page 1-12.

5) International Committee on Taxonomy of Viruses (ICTV). October 2018. Archived from the original on 2019-05-14. Retrieved 2020-01-24.

6) International Committee on Taxonomy of Viruses (ICTV). Retrieved 2020-01-24.

7) Fan Y, Zhao K, Shi ZL, Zhou P (March 2019). Bat Corona viruses in China. Viruses. 11 (3): 210-223.

8) Cherry, James; Demmler-Harrison, Gail J.; Kaplan, Sheldon L.; Steinbach, William J.; Hotez, Peter J. (2017). Feign and Cherry's Textbook of Pediatric Infectious Diseases. Elsevier Health Sciences. p. PT6615. ISBN 978-0-323-39281-5.

9) Woo PC, Huang Y, Lau SK, Yuen KY (2010). Corona virus genomics and bioinformatics analysis. Viruses. 2 (8): $1804-20$.

10) Almeida JD, Berry DM, Cunningham CH, Hamre D, Hofstad MS, Mallucci L, Mclntosh K, Tyrrell DA (November 1968). Virology: Corona viruses. Nature. 220 (5168): 265-278.

11) Definition of Corona virus by Merriam-Webster". Merriam-Webster. Archived from the original on 2020-03-23. Retrieved 2020-03-24.

12) Joseph O. T, Obianime. A. W., Siminialaye M. I., Joseph O. S., Anyanwu C. and Musa T. L. (2019). Clinical study on the effect of Moringa oleifera on serum level of glucose and triglyceride in subjects taken tenofovir, lamivudine and efavirenz combination regimen. European Scientific Journal. Vol.15, (.21). Page 280 -293.

13) Wertheim JO, Chu DK, Peiris JS, Kosakovsky Pond SL, Poon LL (2013). A case for the ancient origin of corona viruses. Journal of Virology. 87 (12): 7039-45.

14) Wertheim JO, Chu DK, Peiris JS, Kosakovsky Pond SL, Poon LL (2014). A case for the ancient origin of corona viruses. Journal of Virology. 87 (12): 7039-45.

15) Novel Corona virus (2019-nCoV) situation reports - World Health Organization (WHO)

16) 2019 Novel Corona virus (2019-nCoV) in the U.S. -. U.S. Centers for Disease Control and Prevention (CDC)

17) Outbreak Notification - National Health Commission (NHC) of the People's Republic of China

18) Novel corona virus (2019-nCoV) - Australian Government Department of Health

19) Jonathan M. Read et al. (2020). Novel corona virus 2019-nCoV: early estimation of epidemiological parameters and epidemic prediction -

20) Maimuna Majumder and Kenneth D. Mandl. (2020). Early Transmissibility Assessment of a Novel Corona virus in Wuhan, China - Harvard University - Computational Health Informatics Program -

21) Imperial College London (2020). Report 3: Transmissibility of 2019-nCoV - 25

22) https://healthwise.punchng.com/covid-19-may-lead-to-high-death-toll-in-africa-melinda-gates/

23) Joseph O. S., Builders M., Joseph O. T., Zubairu S.A., Musa T. and Oyepata p.j. (2019). Sub-acute toxicity study of ethanol leaf extract of Ocimum canum on the kidney of wistar rats. African Journal of Pharmaceutical Research $\&$ Development. Vol. 11 No.1. Page 1-7.

24) Joseph O. S., Builders M., Joseph O. T., Zubairu S.A., Musa T. and Oyepata p.j. (2019). Sub-acute toxicity study of ethanol leaf extract of Ocimum canum on brain, lungs, stomach and spleen of wister rats. African Journal of Pharmaceutical Research \& Development. Vol. 11 No.1. Page 35-42. 


\section{The Dynamics of Differential Impacts of COVID-19 on African Countries Compared to Other Parts of the World}

25) The Guardian. (2020). why Africa is least affected by deaths from covid-19 https://guardian.ng/news/why-africa-isleast-affected-by-deaths-from-covid-19/

26) Joseph O. S., Joseph O. T., Musa T. L and Oyepata P. J. (2019). Histological evaluation of the nephroprotective activity of the ethanol stem extracts of Homalium letestui in Gentamicin - induced albino rats injury, using various staining techniques. Global Scientific Journal. Volume 7, Issue 8. Page 1065-1087.

27) Joseph O.S., Builders M., Emem E.U and Joseph O.T. (2019). EFFECT OF ETHANOL LEAF EXTRACT OF Cassia angustifolia extracts ON LIVER OF WISTER RATS. Global Scientific Journal. Volume 8, Issue 9. Page 1112-11120.

28) Joseph O.S., Builders M., Emem E.U and Joseph O.T. (2019). EFFECT OF ETHANOL LEAF EXTRACT OF Cassia angustifolia extract ON Kidney OF WISTER RATS. Global Scientific Journal. Volume 8, Issue 9. Page 1023-1031.

29) Imperial College London. (2019). Report 3: Transmissibility of 2019-nCoV - 25 January

30) Epidemiology (2019). Case fatality risk of influenza A (H1N1pdm09): a systematic review

31) Chen Want et al (2019). A novel corona virus outbreak of global health concern -. The Lancet.

32) CDC (2005). Symptoms of Novel Corona virus (2019-nCoV).

33) China's National Health Commission news conference on corona virus (2020) - Al Jazeera.

34) Reuters report, January 23,2020 . Wuhan lockdown 'unprecedented', shows commitment to contain virus: WHO representative in China -.

35) Statement on the meeting of the International Health Regulations (2005) Emergency Committee regarding the outbreak of novel corona virus (2019-nCoV) - WHO, January 23, 2020

36) Antonio Villalonga Morales. 2020: Factors that may explain why COVID is less deadly in Africa. Newsletter

37) Gregorio A., Millett MPH, Austin T., Jones MA' David Benkeser MPH, Stefan Baral LM et al. (2020). Differential impacts of COVID-19 on black communities Pages 37-44

38) BABRAHAM INSTITUTE (2020). Charting immune system development in sub-Saharan African

39) Johns Hopkins University COVID-19 Dashboard. 2020. Center for Systems Science and Engineering (CSSE). Johns Hopkins University (2020), Accessed 24th Apr 2020

40) New York State Department of Health COVID-19. 2020. Fatalities. https://covid19tracker.health.ny.gov/views/NYSCOVID19-Tracker/NYSDOHCOVID-19Tracker-Fatalitiesno. Accessed 8th Apr 2020

41) Willis $H$, Williams V. A (2020). Funeral is thought to have sparked a COVID-19 outbreak in Albany, Ga. - and led to many more funerals. The Washington Post, Washington, DC. Page 54-67.

42) Centers for Disease Control and Prevention Corona virus Disease 2019 (COVID-19): Cases in U.S. https://www.cdc.gov/coronavirus/2019-ncov/cases-updates/cases-in-us.html(2020), Accessed 15th Apr 2020

43) Georgia Department of Public HealthCOVID-19 Daily Status Report. https://dph.georgia.gov/covid-19-daily-statusreport (2020), Accessed 9th Apr 2020

44) Helen Thompson. (2012). Early exposure to germs has lasting benefits. Nature. Page 163-176

45) The Guardian. (2020). why Africa is least affected by deaths from covid-19 https://guardian.ng/news/why-africa-isleast-affected-by-deaths-from-covid-19/.

46) Beck G, Habitat GS (1996). Immunity and the Invertebrates. Scientific American. 275 (5):60-66.

47) O'Byrne KJ, Dalgleish AG (2001). Chronic immune activation and inflammation as the cause of malignancy. British Journal of Cancer. 85 (4): 473-83.

48) Litman GW, Cannon JP, Dishaw LJ (2005). Reconstructing immune phylogeny: new perspectives. Nature Reviews. Immunology. 5 (11): 866-79.

49) Restifo NP, Gattinoni L (October 2013). Lineage relationship of effector and memory T cells. Current Opinion in Immunology. 25 (5): 556-63.

50) Kurosaki T, Kometani K, Ise W (March 2015). Memory B cells. Nature Reviews. Immunology. 15 (3): $149-59$. 